\title{
Bile acids cycle disruption in patients with nasopharyngeal carcinoma promotes the elevation of interleukin-10 secretion
}

\author{
Cheng-Shi Wang ${ }^{1}$, Shou-Hou Liu², Jin Peng ${ }^{2}$, Chen Tang ${ }^{2 *}$, Wei-Guo Zhu²*
}

1. Department of Radiotherapy, Lianshui People's Hospital, Huai'an, Jiangsu, 223400, China

2. Department of otorhinolaryngology,Huai'an First People's Hospital ,Nanjing Medical University, Huai'an, Jiangsu, 223300, China

\begin{abstract}
Background: Unclear pathogenesis existed for nasopharyngeal carcinoma.

Aims: to analyze the role of bile acids in the pathogenesis of nasopharyngeal carcinoma.

Methods: 20 healthy volunteers and 20 patients with nasopharyngeal carcinoma were enrolled between January $1^{\text {st }}, 2013$ and December $31^{\text {st }}$, 2014. ESI-QTOF-MS analysis of serum was performed to find altered bile acids components. The biological function of changed bile acids was investigated using in vitro experiment.

Results: Compared with healthy volunteers, the level of DCA and GDCA exhibited higher abundance in patients with nasopharyngeal carcinoma $(\mathrm{p}<0.01)$. Furthermore, the biological function was investigated for the inhibition of DCA and GDCA towards the secretion of IL-10 by CD4+CD25- T cells. Both DCA and GDCA significantly inhibited the secretion of IL-10 by CD4+CD25- T cells. Furthermore, DCA+GDCA can show stronger inhibition towards the secretion of IL-10 than DCA and GDCA.

Conclusion: The inhibition of IL-10 secretion by elevated DCA and GDCA components in nasopharyngeal carcinoma patients is the inducer for nasopharyngeal carcinoma.

Key words: nasopharyngeal carcinoma, interleukin-10 (IL-10), pathogenesis, $\mathrm{T}$ cell, bile acids.

DOI: http://dx.doi.org/10.4314/ahs.v15i4.19

Cite as: Wang C-S, Liu S-H, Peng J, Tang C, ZhuW-G. Bile acids cycle disruption in patients with nasopharyngeal carcinoma promotes the elevation of interlenkin-10 secretion. Afri Health Sci. 2015;15(4):1200-3. bttp:// dx.doi.org/10.4314/abs.v15i4.19
\end{abstract}

\section{Introduction}

Nasopharyngeal carcinoma (NPC) is uncommon in the United States and most countries of the world. Statistical analysis demonstrated the most prevalent of nasopharyngeal carcinoma in Southeast Asians ${ }^{1}$. Diagnosis and therapy methods are relatively poor due to it being relatively rare condition in Western countries. Therefore, elucidation of the pathogenesis of NPC is very important.

\section{* Corresponding authors:}

Chen Tang,

Department of otorhinolaryngology, Huai'an

First People's Hospital ,Nanjing Medical University, Huai'an, Jiangsu, 223300, China.

E-mail: chentanghuaian@163.com

Wei-Guo Zhu,

Department of otorhinolaryngology,Huai'an

First People's Hospital ,Nanjing Medical University, Huai'an, Jiangsu, 223300, China.

E-mail:jshazwg@126.com
Immune function has close relationship with the pathogenesis of NPC. Previous literatures have shown that the appearance of malignant process producing several immunogenic viral proteins within a context of local inflammation and heavy leukocytic infiltration is one major paradox of NPC pathogenesis. Additionally, the different TIL subsets have strong prognostic value for NPC patients ${ }^{2,3}$.

Bile acids are steroid acids predominantly found in bile of mammals. Liver is the major organ responsible for the synthesis of primary bile acids, and the function of bacteria in the colon is to promote the biotransformation of primary bile acids into the secondary bile acids ${ }^{4}$. Bile acids exert many biological functions, including the elimination of cholesterol from the body and driving the flow of bile to eliminate bilirubin. Disruption of bile acids has been reported to be inducers of some diseases, such as diabetes and cancers ${ }^{4}$.

The present study aims to determine the bile acids components in serum and tries to correlate the relationship of bile acids with the pathogenesis of nasopharyngeal carcinoma (NPC). 
Materials and methods

Chemicals antibodies and reagents

CA, DCA, CDCA, GCDCA, GDCA and GCA were purchased from Sigma-Aldrich (St Louis, MO). All other reagents and solvents were of HPLC grade.

\section{Patients and serum preparation}

20 healthy volunteers (age 18-65, body weight $45-90 \mathrm{~kg}$ ) and 20 patients with nasopharyngeal carcinoma (age 17-65, body weight 48-90 kg) were enrolled between January 1 1, 2013 and December 31 ${ }^{\text {st }}, 2014$. Blood was taken, and serum was prepared though the centrifugation at $8,000^{*} \mathrm{~g}$ for $30 \mathrm{~min}$.

\section{Bile acids analysis using UPLC-ESI-QTOFMS}

Both positive and negative modes were used for UPLCESI-QTOFMS running, and the full-scan mode at $\mathrm{m} / \mathrm{z}$ 100-1,000 was employed. Reverse-phase ACQUITY UPLC BEH C18 column $(2.1 \times 50 \mathrm{~mm})$ was used, and the gradient mobile phase was consisted of acetonitrile (A) and water containing $0.2 \%$ formic acid (B). The following gradient was used: $0-0.5 \mathrm{~min}, 100 \% \mathrm{~B}$; 0.5$7.5 \mathrm{~min}, 100 \% \mathrm{~B}-100 \% \mathrm{~A}$. Nitrogen was used as both cone gas $(50 \mathrm{l} / \mathrm{h})$ and desolvation gas $(600 \mathrm{l} / \mathrm{h})$. Source desolvation temperatures were set at $120^{\circ} \mathrm{c}$ and $350^{\circ} \mathrm{c}$, respectively. The capillary voltage and cone voltage was 3000 and $20 \mathrm{~V}$, respectively.

In vitro determination of bile acids' influence towards IL-10 secretion

CD4+CD25- T cells were cultured plated bound anti-CD3 $(5 \mu \mathrm{g} / \mathrm{ml})$ and soluble anti-CD28 $(2 \mu \mathrm{g} / \mathrm{ml})$ antibodies in the presence or absence of bile acids components for $24 \mathrm{~h}$. After the culture, cells were collected, and the protein concentration of IL-10 was determined using ELISA analysis.

\section{Statistical analysis}

The experimental data was presented as mean \pm S.E.M. Comparisons between two groups were performed using a two-tailed unpaired student's $t$ test. A value of $\mathrm{P}<0.05$ was considered to be statistically significant.

\section{Results}

The relative abundance of bile acids was given in Table 1.

Table 1. The relevant abundance (The abundance of bile acids/ the abundance of internal standard) of bile acids components in healthy volunteers and patients with nasopharyngeal carcinoma.

\begin{tabular}{|l|l|l|}
\hline $\begin{array}{l}\text { Bile } \\
\text { components }\end{array}$ & Healthy volunteers & $\begin{array}{l}\text { Patients with nasopharyngeal } \\
\text { carcinoma }\end{array}$ \\
\hline CA & $0.25(0.02)$ & $0.26(0.03)$ \\
\hline GCA & $1.23(0.05)$ & $1.26(0.08)$ \\
\hline DCA & $0.04(0.01)$ & $0.25(0.01)^{* *}$ \\
\hline GDCA & $1.25(0.06)$ & $1.89(0.01)^{* *}$ \\
\hline CDCA & $0.14(0.05)$ & $0.10(0.06)$ \\
\hline GCDCA & $0.89(0.07)$ & $0.95(0.05)$ \\
\hline
\end{tabular}

Compared with healthy volunteers, the level of DCA and GDCA exhibited higher abundance in patients with nasopharyngeal carcinoma $(\mathrm{p}<0.01)$. Futhermore, the biological function was investigated for the inhibition of DCA and GDCA towards the secretion of IL-10 by
CD4+CD25- Tcells. As shown in Figure 1, both DCA and GDCA significantly inhibited the secretion of IL10 by CD4+CD25- T cells. Furthermore, DCA+GDCA can showed stronger inhibition towards the secretion of IL-10 than DCA and GDCA. 


\section{Figure 1}

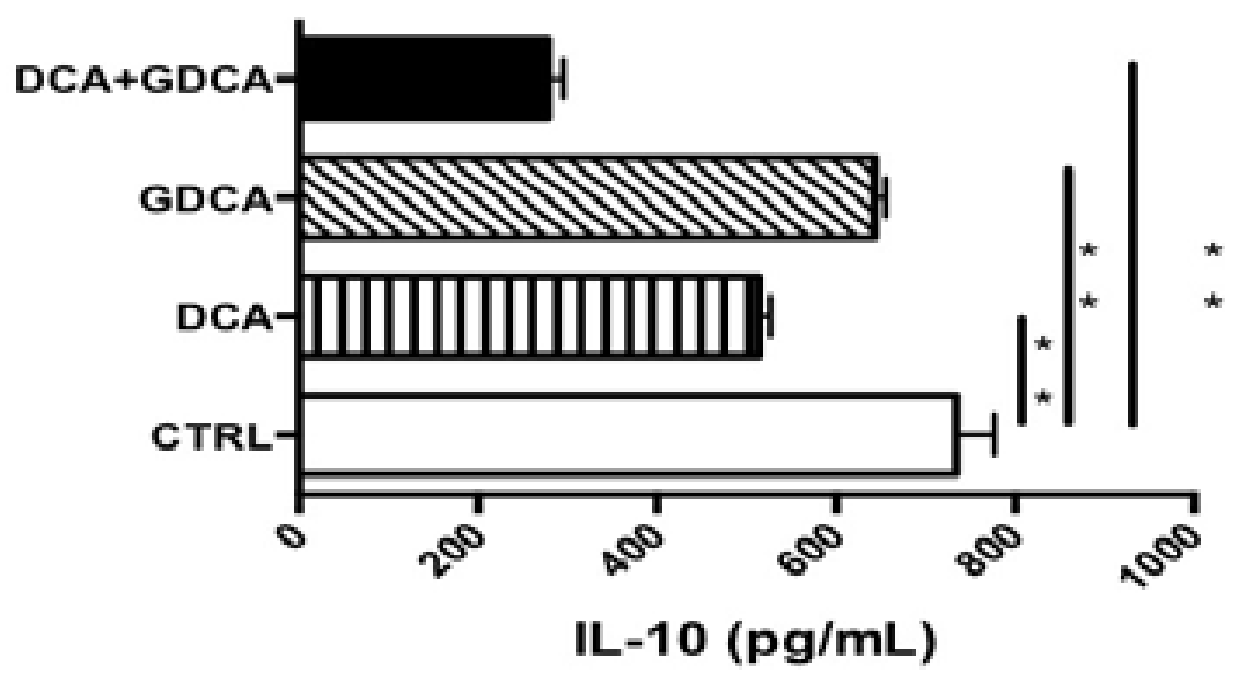

\section{Discussion}

Metabolomics is a technology used to profile the small molecules in biosamples, including serum, urine, and some organ tissues. Metabolomics has been successfully employed to elucidate the pathogenesis of diseases and the toxicity mechanism of xenobiotics. The experiment carried out by Manna et al used metabolomics to elucidate the pathogenesis of colon cancer ${ }^{5}$. Metabolomics has also been applied in the mechanism explanation of dioxin- and diet-induced steatohepatitis in which inflammatory cascade plays a key role ${ }^{6}$.

Bile acids play an important role in the pathogenesis of some diseases. For example, bile acids play an important role in the pathogenesis of obesity and diabetes? The pathogenesis role of bile acids in cancers has also been reported by previous literatures. For example, the dysregulation of bile acids has close relationship with gastrointestinal cancer ${ }^{8}$. Secondary bile acids have been considered to be a cause of colon cancer'. The experiments performed by Liu et al. showed that conjugated bile acids promoted cholangiocarcinoma cell invasive growth through the activation of sphingosine 1-phosphate receptor $2^{10}$.

In this study, we found the role of bile acids in the pathogenesis of nasopharyngeal carcinoma. In patients with nasopharyngeal carcinoma, the level of DCA and GDCA in serum significantly inhibited the secretion of IL-10 protein by T cells. These results will be helpful for the understanding of nasopharyngeal carcinoma's pathogenesis.

\section{References}

1. Lung ML, Cheung AK, Ko JM, Lung HL, Cheng Y, Dai W. The interplay of host genetic factors and Epstein-Barr virus in the development of nasopharyngeal carcinoma. Chin. J. Cancer 2014; 33(11): 556 PubMed -568 .

2. Mrizak D, Martin N, Barjon C, Jimenez-Pailhes AS, Mustapha R, Niki T, Guigay J, Pancre V, de Launoit Y, Busson P, Morales O, Delhem N. Effect of nasopharyngeal carcinoma-derived exosomes on human regulatory T cells. J. Natl. Cancer Inst. 2014; 107(1): 363.

3. Ye SB, Li ZL, Luo DH, Huang BJ, Chen YS, Zhang XS, Cui J, Zeng YX, Li J. Tumor-derived exosomes promote tumor progression and $\mathrm{T}$ cell dysfunction through the regulation of enrichedexosomal microRNAs in human nasopharyngeal carcinoma. Oncotarget 2014; 5(14): 5439 PubMed -5452.

4. Li T, Chiang JY. Bile acids as metabolic regulators. Curr Opin Gastroenterol 2015; 31(2): 159-165.

5. Manna SK, Tanaka N, Krausz KW, Haznadar M, Xue 
X, Matsubara T, Bowman ED, Fearon ER, Harris CC, Shah YM, Gonzalez FJ. Biomarkers of coordinate metabolic reprogramming in colorectal tumors in mice and humans. Gastroenterology 2014; 146(5): 1313 PubMed -1324 .

6. Matsubara T, Tanaka N, Krausz KW, Manna SK, Kang DW, Anderson ER, Luecke H, Patterson AD, Shah YM, Gonzalez FJ. Biomarkers of coordinate metabolic reprogramming in colorectal tumors in mice and humans. Gastroenterology 2014; 146(5): 1313 PubMed $-1324$.

7. Qi Y, Jiang C, Cheng J, Krausz KW, Li T, Ferrell JM, Gonzalez FJ, Chiang JY. Bile acid signaling in lipid metabolism: metabolomics and lipidomic analysis of lipid and bile acid markers linked to anti-obesity and anti-diabetes in mice. Biochim Biophys Acta 2015; 1851(1): 19-29. 8. Tsuei J, Chau T, Mills D, Wan YJ. Bile acid dysregulation, gut dysbiosis, and gastrointestinal cancer. Exp Biol Med 2014; 239 (11): 1489 PubMed -1504.

9. Ajouz H, Mukherji D, Shamseddine A. Secondary bile acids: an underrecognized cause of colon cancer. World J Surg Oncol 2014; 12:164.

10. Liu R, Zhao R, Zhou X, Liang X, Campbell DJ, Zhang X, Zhang L, Shi R, Wang G, Pandak WM, Sirica AE, Hylemon PB, Zhou H. Conjugated bile acids promote cholangiocarcinoma cell invasive growth through activation of sphingosine 1-phosphate receptor 2 . Hepatology 2014; 60(3): 908 PubMed -918. 